\title{
Antiphase Complex-Coupled Surface-Emitting Distributed Feedback Diode Lasers with Absorptive Gratings
}

\author{
Masoud Kasraian, James Lopez, and Dan Botez, Fellow, IEEE
}

\begin{abstract}
Theoretical analysis of antiphase-type complexcoupled, surface-emitting distributed feedback (CC-SE-DFB) diode lasers with absorptive gratings is presented and discussed. Two different designs are studied: one utilizing a semiconductorbased second-order loss and index grating placed at the metal-semiconductor (p-side) interface, and the other employing a combination of metallic and semiconductor materials for the second-order loss and index grating. For certain design parameters, these two types of absorptive-grating structure are shown to select lasing in the symmetric mode (i.e., orthonormal emission in a single-lobe beam pattern). By comparison to metal-grating surface-emitting devices, the threshold gains for these structures are lower by factor of 3 to 4 . For $500-\mu \mathrm{m}$-long gratings, the symmetric-mode is favored to lase with threshold gain values as low as $18 \mathrm{~cm}^{-1}$ and differential quantum efficiency as high as $\mathbf{3 4 \%}$.
\end{abstract}

Index Terms-Absorptive grating, metal grating, second-order complex-coupled distributed-feedback laser, single-lobe beam, surface emitter, 2-D surface emitter.

\section{INTRODUCTION}

C OMPLEX-COUPLED, distributed-feedback (CC-DFB) edge-emitting lasers have recently received considerable theoretical and experimental attention as potential light sources in advanced optical-communication systems [1]-[9]. We have previously reported on the theoretical analysis of both in-phase [10] (i.e., excess gain preferentially placed in the high-index grating regions), second-order, surface-emitting (SE)-CC-DFB as well as antiphase-type [11] (i.e., excess gain preferentially placed in the low-index regions) SE-CCDFB lasers. For antiphase SE-CC-DFB's, we analyzed a relatively simple configuration: a metallic second-order grating placed atop a diode-laser structure [11]. Such structures are of the thin-upper-cladding type [12], [13] and can simply be fabricated by e-beam evaporation and a lift-off process; can operate in a single-lobed, orthonormal beam with a rather uniform near-field intensity pattern, and external differential quantum efficiency $\eta_{D}$ values in excess of $30 \%$. To guarantee symmetric-mode lasing, the metal-grating SE-DFB device must be designed such that the nearest antisymmetric mode experiences more metal absorption losses than the total loss of the symmetric lasing mode due to the radiation outcoupling and metal absorption. Since for an efficient surface-emitting

Manuscript received August 14, 1997; revised September 30, 1997. The authors are with the Department of Electrical and Computer Engineering, University of Wisconsin-Madison, Madison, WI 53706-1691 USA.

Publisher Item Identifier S 1041-1135(98)00432-7. device, high-radiation outcoupling is desirable, relatively large metal absorption losses are unavoidable. As a result of light absorption in the metal grating together with the radiation loss representing the diode surface-emitting output, the (symmetric) lasing mode of metal-grating, SE-DFB lasers has relatively high gain-threshold, $g_{\mathrm{th}}$, values [11] (e.g., $70-80 \mathrm{~cm}^{-1}$ ). In order to significantly reduce $g_{\mathrm{th}}$, it is necessary to make structures for which light absorption in the grating is much less than that for metal-grating devices.

Here, we present the first theoretical treatment of efficient SE-CC-DFB lasers with absorptive gratings. Two different structures are designed and analyzed. We show that antiphase SE-CC-DFB lasers can lase in the symmetric mode (i.e., single-lobed, orthonormal far-field radiation pattern) with a dramatic decrease in gain threshold by comparison to metalgrating devices: from $70-80 \mathrm{~cm}^{-1}$ to $18-20 \mathrm{~cm}^{-1}$; while maintaining relatively high values for the external differential quantum efficiency $\eta_{D}: 30 \%-35 \%$.

\section{ABSORPTIVE-GRATING SE-CC-DFB STRUCTURES}

The longitudinal cross-sections of the two studied SE-CCDFB structures are schematically shown in Figs. 1 and 2. Both designs utilize a multi-quantum-well active region (at $\lambda_{0}=$ $0.98 \mu \mathrm{m})$. The first structure employs a second-order, rectangular, semiconductor-based grating for both feedback and outcoupling mechanisms. The semiconductor-based grating is made of GaAs regions periodically embedded in a p-InGaP cladding layer, and in direct contact with $\mathrm{Au}$, the electricalcontact metal. These GaAs regions incorporate absorbing InGaAs quantum wells that provide the necessary modal gain modulation for favoring the symmetric mode over the antisymmetric one. The grating is in close proximity $(\sim 0.1$ $\mu \mathrm{m})$ of a multiquantum-well (MQW) active-region structure, designed to provide enough gain such that the gain threshold is relatively low ( $\sim 20 \mathrm{~cm}^{1}$. The local modal gain profile (Fig. 1) is a gain/loss grating. All calculations were done as previously by using the method developed by Noll and Macomber [14].

Results of calculations on typical devices, and, for comparison, of the calculations on metal-grating devices [11] are displayed in Table I. For a four-quantum-well (4-QW) separate confinement heterostructure $(\mathrm{SCH})\left(70-\AA \mathrm{In}_{0.20} \mathrm{Ga}_{0.80} \mathrm{As}\right.$ wells, 200- $\AA$ InGaAsP $\left(E_{g}=1.6 \mathrm{eV}\right)$ barriers, $1000 \AA$ InGaAsP waveguiding layers) device with a grating-tooth height of $0.10 \mu \mathrm{m}$, an effective-index step $\Delta n$ of $1.4 \times$ $10^{-2}$ and a $30 \%$ duty cycle (ratio of low-index-region/grating 

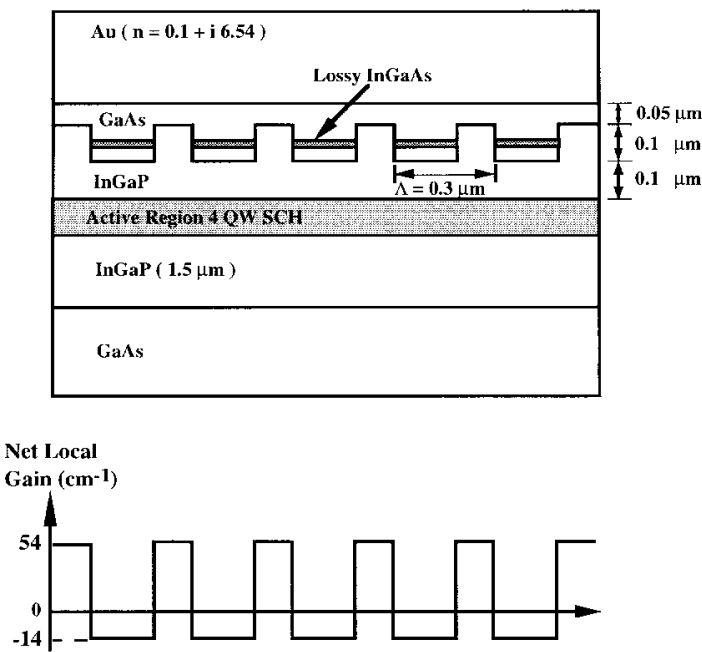

Fig. 1. Schematic diagram of semiconductor-based second-order, surface-emitting antiphase CC-DFB laser structure with absorptive grating. Light generated in the active region is reflected mainly by the low-index Au regions and absorbed primarily in the high-index GaAs-InGaAs regions. Also shown is the calculated net local modal gain profile along the grating.
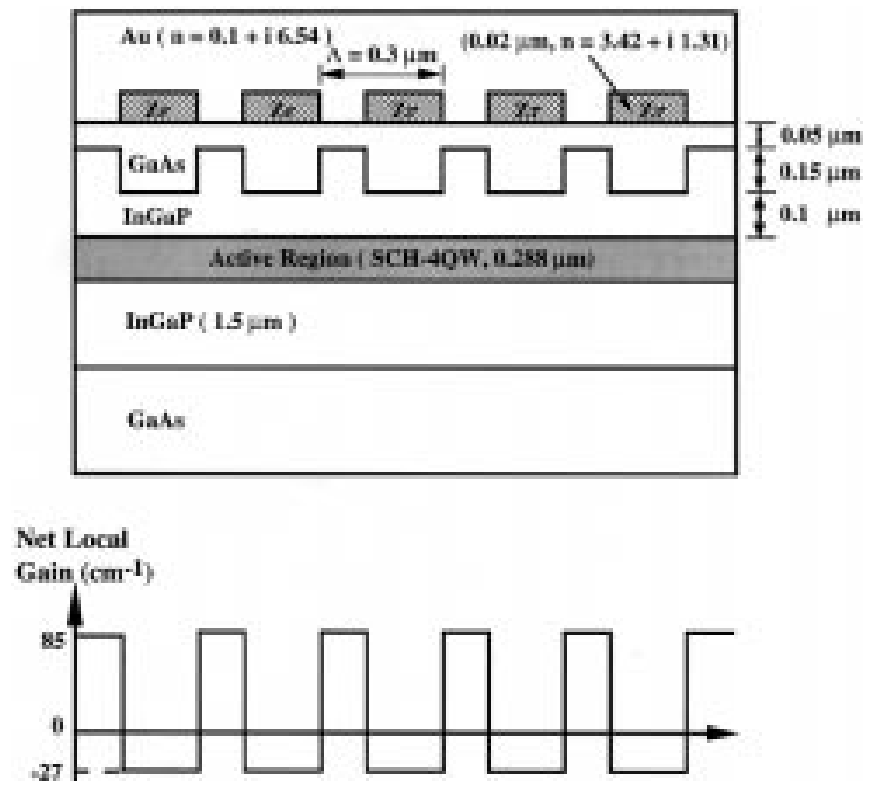

Fig. 2. Schematic diagram of composite semiconductor/metal-grating-based CC-SE-DFB laser with absorptive grating. Also shown is the calculated net local modal gain profile along the grating.

period) the threshold gain for the lasing mode (i.e., the symmetric one) is four times smaller than for the metal-grating case [11]. The intermodal discrimination, effective-index step, and $\eta_{d}$ are similar to those for metal-grating devices. The reason $\eta_{d}$ stays the same is because for metal-grating devices the radiation loss is 3 times higher than for semiconductorgrating devices. This is mainly due to the fact that the difference in the real part of the dielectric constant of the two grating regions (e.g., Au and $\mathrm{Zr}$ ) in the metal grating devices is considerably larger than that in the semiconductor-grating devices. For a different case: 3 -QW active-region, $\Delta n \cong 1.7$ $\times 10^{-2}$, and a $0.10-\mu \mathrm{m}$ grating-tooth height; $\eta_{d}$ is as high
TABLE I

Comparison Between Key Parameters of Optimized Semiconductor-based and Metal-based [11] SECOND-ORDER CC-SE-DFB Lasers with Absorptive Gratings. The Top Row Corresponds to the Device Shown in Fig. 1, While the Middle Row Corresponds to a 3-QW Active-Region Device. $\Delta g_{t h}$ is the Difference in Gain Threshold Between the ANTISymmetric and Symmetric Modes

\begin{tabular}{c|c|c|c|c|c}
\hline & $\kappa$ & I.ength & $\begin{array}{c}\text { Threshold Gain } \\
\mathrm{g}_{\mathrm{th}}\end{array}$ & $\begin{array}{c}\text { Intermodal } \\
\text { Discrimination } \\
\Delta \mathrm{g}_{\mathrm{bh}}\end{array}$ & $\begin{array}{c}\text { External } \\
\text { D.Q.E. } \\
\eta_{\mathrm{b}}\end{array}$ \\
\hline $\begin{array}{c}\text { Semiconductor-grating } \\
\text { DFB3 4QW-SCH }\end{array}$ & $-165-3.8 \mathrm{i} \mathrm{cm}{ }^{-1}$ & $500 \mu \mathrm{m}$ & $18 \mathrm{~cm}^{-1}$ & $6.2 \mathrm{~cm}^{-1}$ & $30 \%$ \\
\hline $\begin{array}{c}\text { Semiconductor-grating } \\
\text { DFB 3QW-SCI }\end{array}$ & $-204-4.6 \mathrm{~cm} \mathrm{~cm}^{-1}$ & $500 \mu \mathrm{m}$ & $21 \mathrm{~cm}^{-1}$ & $4.7 \mathrm{~cm}^{-1}$ & $34 \%$ \\
\hline $\begin{array}{c}\text { Mctal-grating } \\
\text { DFB 2QW-SCH }\end{array}$ & $-35.1-4.4 \mathrm{i} \mathrm{cm}$ & $600 \mu \mathrm{m}$ & $74 \mathrm{~cm}^{-1}$ & $7 \mathrm{~cm}^{-1}$ & $27 \%$ \\
\hline
\end{tabular}

as $39 \%$ (i.e., $30 \%$ higher than in the metal-grating case), and the threshold gain is 3.5 times smaller than for the metalgrating device (for all cases an internal loss coefficient, $\alpha_{i}$, of $3 \mathrm{~cm}^{-1}$ is assumed).

Previously, we have shown [10], [11] that a SE-CC-DFB laser can oscillate in a symmetric mode when the modal-gain difference between the symmetric and antisymmetric modes due to optical-field (longitudinal) overlap with the gain/loss grating [1], [2] overcomes the modal-gain difference based on radiation losses.

The importance of the presence of lossy material in the high-index regions of the semiconductor-based grating for favoring symmetric-mode lasing can be highlighted by comparing two different devices, both having the cross-section shown in Fig. 1 and the same length (e.g., $500 \mu \mathrm{m}$ ), one with lossy and the other with lossless semiconductor grating. As expected, we find that for the lossless semiconductor grating the antisymmetric mode is favored to lase $\left(g_{\mathrm{th}, A}=7 \mathrm{~cm}^{-1}\right)$ while for the lossy grating the symmetric mode is favored to lase $\left(g_{\mathrm{th}, S}=18 \mathrm{~cm}^{-1}\right)$.

While the semiconductor-based grating design demonstrates that anti-phase type, SE-CC-DFB laser can fundamentally favor symmetric mode operation with a dramatic decrease in the gain threshold of the lasing mode (see Table I), the designed structure (Fig. 1) involves an apparently difficult grating to fabricate. As an alternative, we considered another design, depicted in Fig. 2. Here, a composite second-order metallic and semiconductor grating is employed for feedback and outcoupling. The grating, which can be fabricated by standard techniques (i.e., etch and regrowth for the semiconductor grating and liftoff for the metal grating), consists of a periodic variation of a combination of 200 - $\AA$-thick $\mathrm{Zr}$ $\left(n_{\mathrm{Zr}}=3.42+i 1.31\right)$ atop $0.20-\mu \mathrm{m}$-thick GaAs $\left(n_{\mathrm{GaAs}}=\right.$ $3.51)$, and $\mathrm{Au}\left(n_{\mathrm{Au}}=0.1+i 6.54\right)$ atop both $0.05-\mu \mathrm{m}$-thick GaAs and $0.15-\mu \mathrm{m}$-thick InGaP $\left(n_{\mathrm{InGaP}}=3.17\right)$, giving an effective-index step $\Delta n$ of $2 \times 10^{-2}$. $\mathrm{Zr}$ was chosen over other metals (e.g., $\mathrm{Ni}, \mathrm{Ti}, \mathrm{Al}$ ) since it combines a relatively high real part of the refractive index with relatively low optical absorption (at $\lambda_{0}=0.98 \mu \mathrm{m}$ ) [11]. Strong radiation outcoupling can thus occur with relatively small penalty in absorption loss. Although Au has higher optical absorption coefficient than $\mathrm{Zr}$, due to the its extremely low index, light hardly samples $\mathrm{Au}$, while deeply penetrating in the high-index 


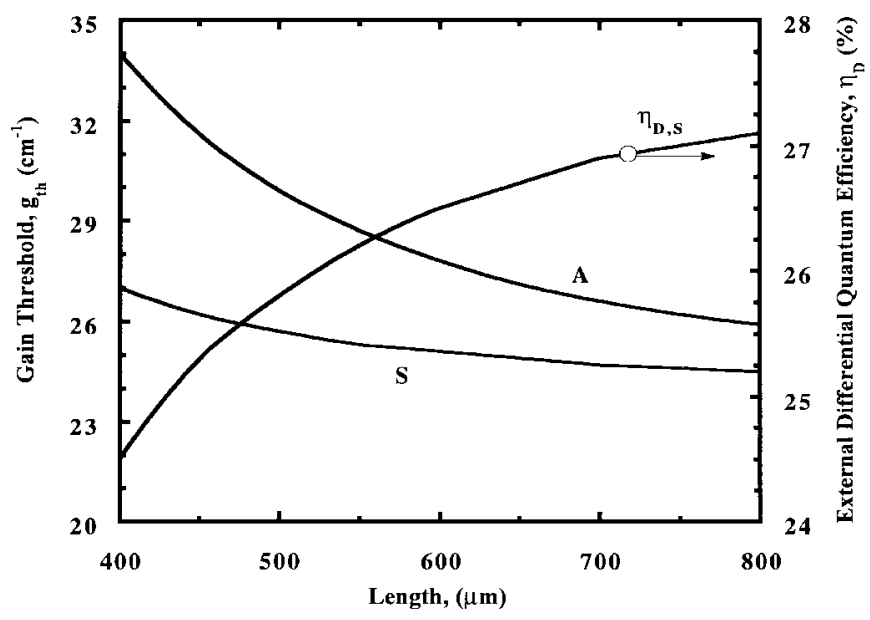

Fig. 3. Gain thresholds of the symmetric (surface-emitting) and antisymmetric modes as well as the external differential quantum efficiency, $\eta_{D}$, versus grating length for the device of Fig. 2.

$\mathrm{Zr}$ regions. Thus an antiphase grating (i.e., high modal gain in the low effective-index regions) is created. The calculated modulation of the local modal gain, shown at the bottom of Fig. 2, is a gain/loss grating.

It should be noted that two transverse modes are supported in the "high-index" regions of this device, an even and an odd mode, just as in antiguided array devices [15]. Overlap integrals between these two modes and the fundamental transverse mode in the low-index region show that over $86 \%$ of the energy couples to the desired mode: the even one, which corresponds to the high effective index. Therefore, we considered in our analysis only the even mode.

Inclusion of the odd mode in the analysis would reduce the feedback between the fundamental mode of the lowindex region as well as alter the Bragg wavelength for the structure (i.e., change the resonance condition) [15]. The reduced feedback will, of course, result in a higher gain threshold. Furthermore, the change in the Bragg wavelength can be considered as a detuning of the grating, hence it will increase the radiation loss coefficient [16]. The net effect on the external differential quantum efficiency would then be negligible.

For the device shown in Fig. 2, we plot in Fig. 3 the gain threshold of the symmetric and antisymmetric modes along with the external differential quantum efficiency, $\eta_{D}$, of the symmetric mode as a function of grating length. While the symmetric mode is clearly favored to lase over the entire range of grating lengths, there is also a tradeoff between $\eta_{D}$ and intermodal discrimination. For longer lengths the efficiency is best but the intermodal discrimination may not be enough to prevent multimode operation at high drive levels above threshold. Therefore, it is necessary to use a shorter length device. For example, for $L=450 \mu \mathrm{m}$ the $\eta_{D}$ is $\sim 25 \%$ and the intermodal discrimination is $5.5 \mathrm{~cm}^{-1}$. The gain threshold of the symmetric mode in this case is $26 \mathrm{~cm}^{-1}$. When comparing this structure to the metal-grating DFB structure (Table I) we see that, while the $\eta_{D}$ values are comparable, the gain threshold of the lasing mode for the semiconductor-metal- grating device is nearly a factor of 3 lower, which results in a more overall efficient device.

\section{CONCLUSION}

We show, for the first time, that efficient, single-lobe surface-emitting devices can be made by using second-order CC-DFB structures with absorptive gratings. The key is to design a semiconductor-based complex-coupled grating that will both favor oscillation in the symmetric mode as well as provide low gain threshold. Thus, one can achieve relatively low gain thresholds $\left(\sim 20 \mathrm{~cm}^{-1}\right)$ with moderately high external differential quantum efficiency (30\%-35\%). Ridgeguide devices should radiate in a single spatial mode up to 50-100-mW CW, definitely higher than vertical-cavity surface emitters ( $\sim 1-\mathrm{mW}$ CW single mode). Two-dimensional (2-D) surface-emitting devices, combining the CC-DFB grating with a resonant antiguided phase-locked array [15], are likely to provide watts of stable, coherent power.

\section{REFERENCES}

[1] K. David, J. Buus, and R. G. Baets, "Basic analysis of AR-coated, partly gain coupled DFB lasers: The standing wave effect," IEEE J. Quantum Electron., vol. 28, pp. 427-433, 1992.

[2] R. G. Baets, K. David, and G. Morthier, "On the distinctive features of gain coupled DFB lasers and DFB lasers with second-order grating," IEEE J. Quantum Electron., vol. 29, pp. 1792-1798, 1993.

[3] Y. Nakano, Y. Luo, and K. Tada, "Facet reflection independent, single longitudinal mode oscillation in a GaAlAs/GaAs distributed feedback laser equipped with a gain-coupling mechanism," Appl. Phys. Lett., vol. 55, pp. 1606-1608, 1989.

[4] K. Kudo, S. Aria, and K. C. Shin, "The optical gain coupling saturation effect on the linewidth property of complex-coupled DFB lasers," IEEE Photon. Technol. Lett., vol. 6, pp. 482-485, 1994.

[5] W. T. Tsang, F. S. Choa, M. C. Wu, Y. K. Chen, R. A. Logan, S. N. G. Chu, A. M. Sergent, and C. A. Burrus, "Semiconductor distributed feedback lasers with quantum well or superlattice gratings for index or gain-coupled optical feedback," Appl. Phys. Lett., vol. 60 , pp. 2580-2582, 1992.

[6] J. Zoz, T. W. Johannes, A. Rast, B. Borchert, U. Barabas, and W. Harth, "Dynamics and stability of complex-coupled DFB lasers with absorptive gratings," IEEE J. Quantum Electron., vol. 31, pp. 1432-1442, 1996.

[7] T. Makino, H. Lu, and J. D. Evans, "Temperature dependence of the threshold current in gain-coupled distributed feedback lasers with periodically etched quantum wells: Mechanism for an appearance of infinite $T_{0}$, , Appl. Phys. Lett., vol. 68, pp. 1892-1894, 1996.

[8] A. Rast, T. W. Johannes, W. Harth, and G. Franz, "New complexcoupled DFB-laser with a contacted surface grating for $\lambda=1.55 \mu \mathrm{m}$," Proc. Inst. Elect. Eng.-Optoelectron., vol. 142, pp. 162-164, 1995.

[9] M. L. Osowski, R. Panepucci, I. Adesida, and J. J. Coleman, "A strainedlayer InGaAs-GaAs asymmetric cladding gain-coupled DFB laser with titanium surface gratings by metalorganic chemical vapor deposition," IEEE Photon. Technol. Lett., vol. 9, pp. 422-424, 1997.

[10] M. Kasraian and D. Botez, "Single-lobed far-field radiation pattern from surface-emitting complex-coupled distributed-feedback diode lasers," Appl. Phys. Lett., vol. 67, pp. 2783-2785, 1995.

[11] M. Kasraian and D. Botez, "Metal-grating-outcoupled, surface-emitting distributed-feedback diode lasers," Appl. Phys. Lett., vol. 69, pp. 2795-2797, 1996.

[12] C. H. Wu, P. S. Zory, and M. A. Emanuel, "Contact reflectivity effects on thin p-clad InGaAs single quantum-well lasers," IEEE Photonics. Technol. Lett., vol. 6, pp. 1427-1429, 1994.

[13] G. M. Smith, D. V. Forbes, R. M. Lammert, and J. J. Coleman, "Metallization to asymmetric cladding separate confinement heterostructure lasers," Appl. Phys. Lett., vol. 67, pp. 3847-3849, 1995.

[14] R. J. Noll and S. H. Macomber, "Analysis of grating surface emitting lasers," IEEE J. Quantum Electron., vol. 26, pp. 456-466, 1990.

[15] D. Botez and D. R. Scifres, Eds., Diode Laser Arrays. Cambridge, U.K.: Cambridge Univ. Press, 1994, pp. 1-72.

[16] G. Anders, M. Larsson, M. Eriksson, and M. Hagberg, "Highly efficient grating coupled surface emitters," presented at the Annu. Meet. Opt. Soc. Amer., 1996, paper WII3. 